\title{
Motor evoked potentials of the respiratory muscles in tetraplegic patients
}

\author{
Mark A Lissens and Guy G Vanderstraeten \\ Department of Physical Medicine and Rehabilitation, University Hospital Ghent, Belgium
}

\begin{abstract}
We studied the respiratory muscles with magnetic transcranial stimulation (TCS) in four spinal cord injured (SCI) patients as compared to age-matched controls from a database of 40 healthy subjects. These SCI patients all had spinal cord lesions above C6 level with a clinically incomplete tetraplegia. One patient was artificially ventilated. Motor evoked potentials (MEPs) were recorded from the diaphragm, the scalenes, the parasternal intercostals and the expiratory rectus abdominis during inspiration and expiration.

In patients with incomplete tetraplegia MEP latency times were significantly prolonged in the scalenes and the parasternal intercostals, both during inspiration and expiration, and were nearly normal for the diaphragm, which was found to be more or less preserved. The mean MEP amplitudes in these patients for all inspiratory muscles studied were significantly decreased in tetraplegic patients, in part due to a decreased number of innervating axons and muscle hypotrophy. No MEPs could be obtained from the abdominal muscles, except in one C3 tetraplegic patient, in whom only a very small response was seen during expiration, with a very delayed latency time. The much lower location of their innervating nerve roots (T10) and the much longer distance of their spinal exit zone from the level of injury at the cervical spinal cord might at least partially explain this phenomenon. In the ventilator-dependent tetraplegic patient no MEPs could be obtained from any of the muscles studied.

Thus, magnetic TCS is a painless and easily applicable technique to investigate the central motor conduction properties of the respiratory muscles, both in healthy humans and in tetraplegic patients.
\end{abstract}

Keywords: respiratory muscles; motor evoked potentials; magnetic transcranial stimulation; motor cortex; diaphragm; scalenes

\section{Introduction}

Previously, the diaphragm was thought to be the only important contracting muscle during quiet breathing in humans. Now it is known that the parasternal intercostal muscles and also the scalenes, conventionally regarded as accessory muscles of inspiration, are also primary inspiratory muscles. ${ }^{8-10,11,26}$ The abdominal muscles (ie the abdominal oblique, the rectus and transversus abdominis muscles) and the transversus thoracis muscle (also called triangularis sterni or sternocostalis muscle) are active during quiet expiration in tetraplegic subjects ${ }^{24}$ and in the sitting and standing position also in healthy humans, ${ }^{28}$ making spontaneous quiet expiration an active process instead of a purely passive maneuver as formerly thought. $5,12,13,23,28$ Moreover, it is known that in tetraplegic patients the behaviour of the respiratory muscles, and consequently the motion of the rib cage and abdomen is changed. ${ }^{24}$ However, the characteristics of central motor conduction as well as the

Correspondence: Dr. MA Lissens, M.D., Department of Physical Medicine and Rehabilitation, University Hospital of Ghent, De Pintelaan, 185, B-9000 GHENT, Belgium properties of the corticospinal tract of the respiratory muscles has never previously been studied in patients with tetraplegia due to a high cervical spinal cord lesion.

Magnetic transcranial stimulation (TCS) of the motor cortex has been shown to be a non-invasive and painless technique to study the integrity of the corticospinal tracts ${ }^{2}$ and has already several clinical applications. ${ }^{6,21}$ Magnetic stimulation allows safe, easy and effective stimulation of most neural structures, unimpeded by fat and bone and without discomfort to the patient. Compound motor evoked potentials (MEPs) from various muscles can be obtained in response to magnetic transcranial motor cortex stimulation and nerve root, plexus and peripheral nerve stimulation. When subtracting the latency time after nerve root stimulation from the cortical latency time, one can calculate the central motor conduction time (CMT).

Up to now, the respiratory muscles (diaphragm, scalenes, intercostals, sternocleidomastoid and abdonimals) were only investigated with electrical TCS and only in a small number of healthy subjects. For the 
diaphragm a rather invasive recording technique through a gastro-oesophageal catheter was used. Only a very limited number of subjects were studied (three, seven and five subjects in the studies conducted by Gandevia et al in 1987, 1988 and 1990, respectively. ${ }^{16-18}$ However, a systematic study on magnetic TCS of the respiratory muscles had not yet been published. We reported a painless and noninvasive technique using magnetic transcranial brain stimulation and surface recording electrodes to investigate the central motor conduction properties of the diaphragm, the principal inspiratory muscle ${ }^{19,29}$ and the abdominal muscles involved in expiration. ${ }^{23}$ The present study is designed to explore the behaviour of the primary inspiratory muscles and of the abdominals when the motor cortex is transcranially stimulated during the different phases of the respiratory cycle, as well in healthy humans as in patients with tetraplegia. We investigated the age-related and sex-related differences of CMCT and amplitude of the MEP after cortical and nerve root stimulation. Taking into account that pre-activation of the target muscle increases the response amplitude and reduces the response latency time by some 1-2 ms, measurement of the MEPcharacteristics will allow us to determine the corticospinal conduction properties of the respiratory muscles and to describe the MEP differences during inspiration and expiration. Moreover, magnetic transcranial motor cortex stimulation can be helpful to find out whether the rhythmic activation of the respiratory muscles, effected at a supraspinal site and depending on a central involuntary mechanism, is mediated through a common neural pathway, or through different parallel pathways activated all together, as well as to know whether the MEPparameters, corticospinal conduction properties, and neurocontrol are different in the inspiratory and expiratory muscles, and in tetraplegic patients as compared to healthy subjects.

\section{Materials and methods}

We studied the respiratory muscles in 40 healthy subjects, 20 males and 20 females, divided into four age decades between 20 and 60 years. Their mean age was $38.95 \pm 11.99$ (SD) years. In addition, four spinal cord injured patients, two women and two men aged 40, 22, 44 and 18 years, with lesions above C6 level were examined. The level of their injury was located at C2, C3 (two patients) and C5. They were examined at $9,16,10$ and 8 months postinjury, respectively. They presented with a clinically incomplete lesion, but one patient (with a spinal cord lesion at $\mathrm{C} 3$ level) was artificially ventilated.

TCS was delivered through a circular coil (outer diameter $12.5 \mathrm{~cm}$ ) by a Magstim 200 magnetic stimulator (Magstim company, Whitland, UK), with a maximum output of 2.5 tesla. MEPs were recorded with $0.5 \mathrm{~cm} \mathrm{Ag-AgCl}$ cup electrodes at the three primary inspiratory muscles (ie the diaphragm, the scalenes, the parasternal intercostals) and the expiratory rectus abdominis. For the diaphragm the active electrode was placed at the xiphoid process, and the reference electrode on the lower border of the rib cage at midclavicular level. ${ }^{22,29}$ For the parasternal intercostal muscles the active electrode was placed at the third intercostal space directly parasternally, and the reference electrode at the second rib. For the scalenes the active electrode was placed approximately $2 \mathrm{~cm}$ above the clavicle in the supraclavicular triangle at midclavicular level and the reference electrode at the clavicle. For the rectus abdominis muscle the active electrode was placed at the umbilicus level $2 \mathrm{~cm}$ lateral to the midline and the reference electrode $2 \mathrm{~cm}$ above the active one. The ground electrode was placed at the acromion for the scalenes, at the manubrim sterni for the parasternal intercostal muscles, at the sternum for the diaphragm, and at the iliac crest for the abdominal muscles. Magnetic TCS was applied with the center of the coil above $\mathrm{Cz}$, according to the international 10-20 system for placement of EEGelectrodes. Subsequently, the cervical and thoracic nerve roots were stimulated at C4-C5, T2-T3 and T9$\mathrm{T} 10$, in order to calculate the CMCT, ie the difference between the latency times of cortical and nerve root stimulation. Magnetic transcranial motor cortex stimulation as well as nerve root stimulation were performed at the end of the inspiratory and expiratory phase (monitored through the electromyographic signals of the recorded muscles). All stimuli were delivered two to three times, and the best MEP (ie with the largest amplitude) was taken into account, during inspiration and expiration as well as after cortical and nerve root stimulation.

The muscle responses or motor evoked potentials (MEPs) were registered on a four-channel electromyograph (Medelec/Teca Sapphire Premiere, Vickers Medical, Woking, Surrey, England) with a filter setting of $3 \mathrm{~Hz}$ to $10 \mathrm{kHz}$. Sweep speed was set at $10 \mathrm{~ms}$ per division or a total sweep duration of $100 \mathrm{~ms}$. MEP latency times and amplitudes were measured, and the CMCT was calculated. MEP amplitudes after cortical stimulation were measured from peak to peak. MEP latency times after cortical and nerve root stimulation were measured at the onset of the first deflection of the MEP.

All parameters were statistically analysed with nonparametric methods, and according to the instructions published in the New England Journal of Medicine $^{1}$ and the British Medical Journal. ${ }^{19}$

\section{Results}

All results are summarized in Table 1. After cortical stimulation on MEP latency times increase from the scalenes to the abdominal muscles, both during inspiration (scalenes: $8.31 \pm 1.24 \mathrm{~ms}$, parasternal intercostals: $10.08 \pm 1.47 \mathrm{~ms}$, diaphragm: $15.46 \pm 0.97 \mathrm{~ms}$, and rectus abdominis $20.88 \pm 1.65 \mathrm{~ms}$ ) and expiration 
Table 1

\begin{tabular}{lccrrrr}
\hline \multicolumn{1}{c}{ Muscle } & Lat. $(\mathrm{ms})$ & \multicolumn{1}{c}{$\begin{array}{c}\text { Inspiration } \\
\text { Ampl. }(\mu \mathrm{V})\end{array}$} & \multicolumn{1}{c}{ CMCT $(\mathrm{ms})$} & \multicolumn{1}{c}{ Lat. $(\mathrm{ms})$} & \multicolumn{1}{c}{\begin{tabular}{c} 
Axpiration \\
\cline { 5 - 7 }$(\mu \mathrm{mpl})$
\end{tabular}} & \multicolumn{1}{c}{ CMCT $(\mathrm{ms})$} \\
\hline Scalenes & $8.31 \pm 1.24$ & $2385.9 \pm 1994$ & $5.28 \pm 1.01$ & $9.71 \pm 1.198$ & $1724.1 \pm 1763$ & $6.49 \pm 1.17$ \\
Parasternal intercostals & $10.076 \pm 1.467$ & $1293.0 \pm 1469$ & $6.356 \pm 1.24$ & $11.423 \pm 1.40$ & $811.1 \pm 1084$ & $7.42 \pm 1.27$ \\
Diaphragm & $15.46 \pm 0.97$ & $2530.3 \pm 1831$ & $7.69 \pm 0.95$ & $16.94 \pm 0.99$ & $1625.1 \pm 1384$ & $8.558 \pm 1.06$ \\
Rectus abdominus & $20.88 \pm 1.65$ & $663.7 \pm 554.7$ & $13.22 \pm 1.61$ & $18.85 \pm 1.52$ & $1112.9 \pm 838.0$ & $11.76 \pm 1.49$ \\
\hline
\end{tabular}

Mean values \pm standard deviations of the MEP-latency time, MEP-amplitude and CMCT during inspiration and expiration for the scalenes, parasternal intercostals, diaphragm and rectus abdominis muscles in 40 healthy subjects

(scalenes: $9.71 \pm 1.20 \mathrm{~ms}$, parasternal intercostals: $11.42 \pm 1.40 \mathrm{~ms}$, diaphragm: $16.94 \pm 0.99 \mathrm{~ms}$ and rectus abdominis: $18.85 \pm 1.52 \mathrm{~ms}$ ). Central motor conduction times are within the same range for all three inspiratory muscles, both during inspiration (scalenes: $5.28 \pm 1.01 \mathrm{~ms}$, parasternal intercostals: $6.36 \pm 1.24 \mathrm{~ms}$, diaphragm: $7.69 \pm 0.95 \mathrm{~ms}$ ) and expiration (scalenes: $6.49 \pm 1.17 \mathrm{~ms}$, parasternal intercostals: $7.42 \pm 1.27 \mathrm{~ms}$, diaphragm: $8.56 \pm 1.06 \mathrm{~ms}$ ), but increase slightly from the scalenes to the diaphragm. Conversely, the abdominal or expiratory muscles show a much longer CMCT, notably $13.22 \pm 1.61 \mathrm{~ms}$ at inspiration and $11.76 \pm 1.49 \mathrm{~ms}$ at expiration. MEP amplitudes are largest in the diaphragm $(2530 \pm 1831 \mu \mathrm{V})$ and scalenes $(2385 \pm 1994 \mu \mathrm{V})$ and smaller in the parasternal intercostal $(1293 \pm 1469 \mu \mathrm{V})$ and abdominal (1112 \pm $838 \mu \mathrm{V}$ ) muscles (see also Figure 1).

The abdominal cortical MEP-latency time and amplitude in healthy subjects were statistically significantly correlated with age. The cortical MEPlatency time of the rectus abdominis increased with age $\left(r_{\mathrm{s}}=0.4064 ; P=0.009\right)$. The abdominal MEPamplitude decreased with age $\left(r_{\mathrm{s}}=-0.4474\right.$; $P=0.004)$. The abdominal CMCT as well as all other parameters of the inspiratory muscles did not show any significant correlation with age.

According to the Wilcoxon matched-pairs signedrank test highly statistically significant differences $(P<0.001)$ were found between inspiration and expiration in all muscles studied and for all parameters, both in males and females.

Most variables did not show any significant sex differences.

MEPs from the respiratory muscles in incomplete tetraplegic patients are illustrated in Figure 2. The mean MEP latency times in the three examined patients with incomplete tetraplegia were $10.6 \mathrm{~ms}$ (normal $8.31 \pm 1.24$ ) during inspiration, and $11.97 \mathrm{~ms}$ (normal 9.71 \pm 1.20 ) during expiration for the scalenes; $12.5 \mathrm{~ms}$ (normal 10.08 \pm 1.47 ) during inspiration, and $14.73 \mathrm{~ms}$ (normal 11.42 \pm 1.40 ) during expiration for the parasternal intercostals; $14.7 \mathrm{~ms}$ (normal 15.46 \pm 0.97 ) during inspiration, and $17.3 \mathrm{~ms}$ (normal 16.94 \pm 0.99 ) during expiration for the diaphragm.

The mean MEP amplitudes in these patients were $1781 \mu \mathrm{V}$ (normal 2386) during inspiration and $1012 \mu \mathrm{V}$ (normal $1724 \mu \mathrm{V}$ ) during expiration for the

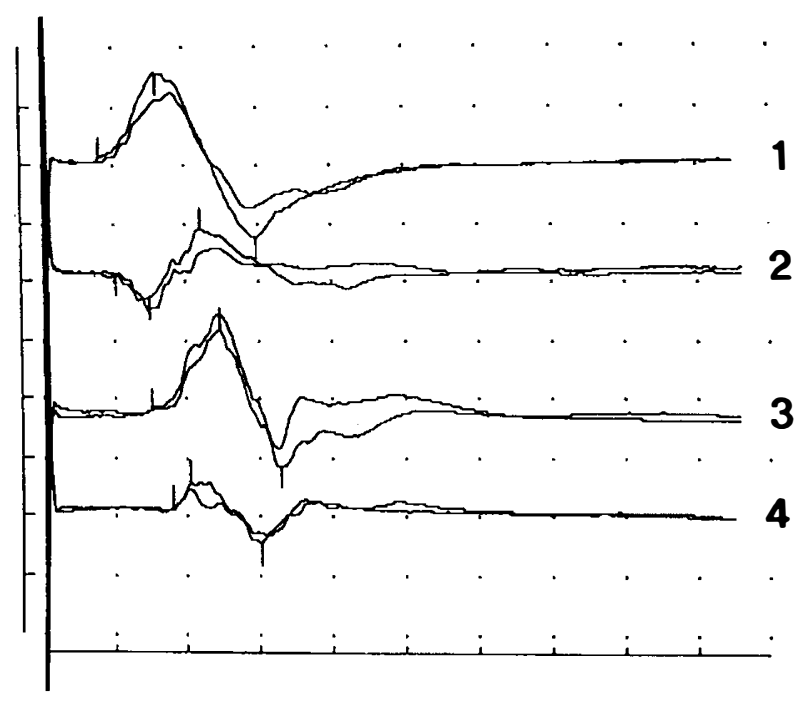

Figure 1 MEPs from the respiratory muscles in a healthy subject. Calibrations are $2 \mathrm{mV}$ per division, and $100 \mathrm{~ms}$ sweep duration or $10 \mathrm{~ms}$ per division. $1=$ scalenes, $2=$ parasternal intercostals, $3=$ diaphragm, $4=$ rectus abdominis

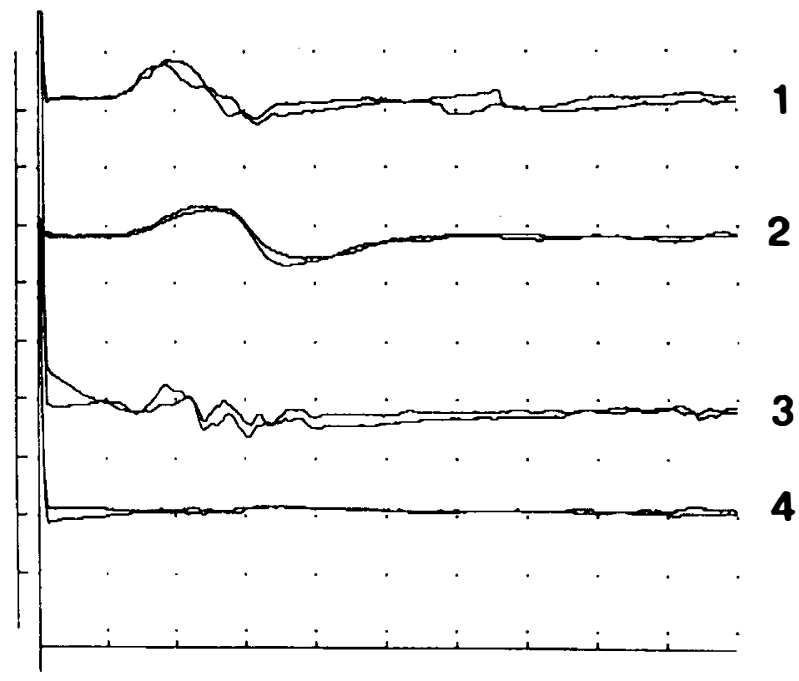

Figure 2 MEPs from the respiratory muscles in a patient with incomplete tetraplegia (C3). Calibrations are $1 \mathrm{mV}$ per division for the upper two traces and $500 \mu \mathrm{V}$ for the lower two traces. Sweep duration is $100 \mathrm{~ms} 10 \mathrm{~ms}$ per division. $1=$ scalenes, 2 =parasternal intercostals, $3=$ diaphragm, $4=$ rectus abdominis 
scalenes, $390 \mu \mathrm{V}$ (normal $1293 \mu \mathrm{V}$ ) during inspiration, and $343 \mu \mathrm{V}$ (normal $811 \mu \mathrm{V}$ ) during expiration for the parasternal intercostals, $374 \mu \mathrm{V}$ (normal $2530 \mu \mathrm{V}$ ) during inspiration and $124 \mu \mathrm{V}$ (normal $1625 \mu \mathrm{V}$ ) during expiration for the diaphragm.

The CMCT could be measured in only one subject with an incomplete $\mathrm{C} 5$ lesion, because cervical root stimulation was impossible, or contraindicated in the other subjects. In this one case CMCT during inspiration was found to be $7.05 \mathrm{~ms}, 8.65 \mathrm{~ms}$ and $7.45 \mathrm{~ms}$ for the scalenes, parasternal intercostals and diaphragm, respectively. During expiration these values were $9.85 \mathrm{~ms}, 13.0 \mathrm{~ms}$ and $9.2 \mathrm{~ms}$, respectively.

No MEPs could be obtained from the abdominal muscles, except in one incomplete $\mathrm{C} 3$ tetraplegic patient, in whom only a very small response was seen during expiration with a latency time of $26.9 \mathrm{~ms}$ (compared to $18.7 \pm 1.3 \mathrm{~ms}$ in controls), and an amplitude of $40 \mu \mathrm{V}(886 \pm 440 \mu \mathrm{V}$ in controls).

In the ventilator-dependent tetraplegic patient no MEPs could be obtained from any of the muscles studied.

\section{Discussion}

It can be concluded that magnetic transcranial brain stimulation activates motor nuclei of all examined respiratory muscles via rapidly conducting mono- or oligosynaptic pathways from the motor cortex to the human respiratory motoneurons, similarly as has been demonstrated for the corticospinal pathways to the limb muscles. ${ }^{14,16,22}$ Our findings confirm that the motor cortex can access truncal motoneuron pools during respiratory maneuvers, as was already shown by Gandevia et al in 1987 and $1988 .^{16,17}$ The increase in latency time from the scalenes to the abdominal muscles could be expected taking into consideration the distance of the respective muscles from the motor cortex. This was also demonstrated by Gandevia et al in $1988,{ }^{17}$ who found a relationship between the distance from the motor cortex to the spinal exit zone of the motor roots and the latency time. Conversely, CMCT of the abdominal or expiratory muscles is much longer, notably $13.22 \pm 1.61 \mathrm{~ms}$ at inspiration and $11.76 \pm 1.49 \mathrm{~ms}$ at expiration. This might reflect their longer distance from the motor cortex and the lower location of their motoneurons in the medullary anterior horn (at T10 level for the umbilical rectus abdominis muscle fibers). Compared to the studies by Gandevia et al in $1988,{ }^{17}$ who investigated the (3rd/ 4th) parasternal intercostals in seven subjects with electrical TCS, our cortical MEP latency times and $\mathrm{CMCT}$ are consistent with their MEP latency time and CMCT, although our CMCT is somewhat longer $(0.56$ to $1.62 \mathrm{~ms}$ ). This was to be expected, since latency times after magnteic TCS are known to be a few ms longer than after electrical TCS, 2,3,7,27 due to the different localisation of the initially stimulated structures through cortico-cortical connections. On the other hand inhibitory and facilitating mechanisms have an important influence in magnetic TCS. Similarly, we found for the diaphragm latency times that were $3-4 \mathrm{~ms}$ longer (15.46 $\mathrm{ms}$ during inspiration and 16.94 during expiration) than those reported by Gandevia et al in $1987^{16}$ (12.3 ms). However, they studied only three subjects with electrical TCS, and used a gastro-oesophageal catheter to record the MEPs.

Standard deviations for both MEP latency times and CMCT are very small in all respiratory muscles studied. The small variability can be explained by the probable absence of any relay in the pontomedullary respiratory centers, or by a lower number of central connections. ${ }^{20}$ This makes the latency times and CMCTs very reliable parameters in the diagnosis and follow-up of neurological and respiratory disorders affecting the central motor conduction properties of the respiratory muscles.

MEP amplitudes were largest in the diaphragm $(2530 \pm 1831 \mu \mathrm{V})$ and scalenes $(2385 \pm 1994 \mu \mathrm{V})$, and smaller in the parasternal intercostal $(1293 \pm$ $1469 \mu \mathrm{V})$ and abdominal $(1112 \pm 838 \mu \mathrm{V})$ muscles. In contrast to the MEP latency times and CMCT, MEP amplitudes all showed large standard deviations. This large variability can be at least partially explained by the fact that the amplitude depends on several local factors, such as skin thickness and underlying layers of fat and connective tissue, as well as on interindividual differences of abdominal, thoracic and rib cage configuration and volume. Morover, it is known that MEP-amplitudes show a much larger variability, as compared to the more stable latency times. ${ }^{7,22}$ The largest amplitudes were seen in the most superficially located muscles, ie the (crural) diaphragm and scalenes. The other two muscles are situated much deeper; the abdominal muscles are covered with thicker layers of skin and soft tissues such as fat and connective tissue, and the parasternal intercostals are positioned between the ribs and sternum. Moreover, the intercostal muscles contain less muscle fibers as compared to the scalenes, diaphragm and abdominal muscles.

Cortical MEP-latency times, CMCT and amplitudes of all inspiratory muscles (scalenes, parasternal intercostals, and diaphragm) as well as the abdominal CMCT showed no significant correlation with age. Conversely, the abdominal cortical MEP-latency time and amplitude were statistically significantly correlated with age. The cortical MEP-latency time of the rectus abdominis increased with age $\left(r_{\mathrm{s}}=0.4064 ; P=0.009\right)$. The abdominal MEP-amplitude decreased with age $\left(r_{\mathrm{s}}=-0.4474 ; P=0.004\right)$. Slowing of nerve conduction velocity with increasing age has been found in peripheral motor and sensory nerves, especially in subjects over 60 years of age. ${ }^{4,15,25}$ In contrast to the nerves of the upper and especially lower limbs, the respiratory muscles, and certainly the inspiratory muscles are located more proximally. This could account for the absence of any correlation with age 
of the MEP-latency times and amplitudes of the primary inspiratory muscles (diaphragm, parasternal intercostals and scalenes). For the abdominal muscles on the other hand we found age correlations very similar to those seen for other peripheral sensory and motor nerves: there was an increase of the cortical MEP-latency time of the rectus abdominis and a decrease of the abdominal MEP-amplitude with advancing age. This could be explained by the more distal location of the abdominal muscles, and by the longer course of their innervating nerves. The CMCT of the rectus abdominis muscle did not show any correlation with age in subjects under 60. This supports the hypothesis that central components of the nervous system mature and age at a slower rate than peripheral components. Since we used surface electrodes to record the compound muscle action potentials, the decline in MEP-amplitude of the abdominal muscles probably also results from a thickening abdominal layer of subcutaneous fat and connective tissue, and an increasing elongation or distension of collagen fibers with age.

Most parameters of the respiratory muscles studied are not sex-related, as we have already shown for the diaphragm and abdominal muscles in a smaller population, ${ }^{22,23}$ and confirmed in a larger population for the diaphragm by Zifko et al. ${ }^{29}$

According to the Wilcoxon matched-pairs signedrank test highly statistically significant differences $(P<0.001)$ were found between inspiration and expiration in all muscles studied and for all parameters, notably cortical MEP-latency time, MEP-amplitude and CMCT, both in males and females and in healthy as well as in tetraplegic subjects. In summary, the MEP-latency time and CMCT were significantly longer, and the MEPamplitude decreased significantly during expiration as compared to inspiration in all three inspiratory muscles, ie scalenes, parasternal intercostals and diaphragm. Exactly the opposite was seen in the rectus abdominis muscle, where the MEP-latency time and CMCT shortened significantly, and the MEP-amplitude increased during expiration as compared to inspiration. Taking into account that pre-activation of the target muscle increases the response amplitude and reduces the response latency time by some $1-2 \mathrm{~ms},{ }^{27}$ one can assume that contraction of the respiratory muscles during either the inspiratory or expiratory phase will be reflected in their MEP-latency time, CMCT and MEPamplitude. Consequently, this will not only allow us to determine the corticospinal conduction properties of the respiratory muscles, but also to describe the MEP differences during inspiration and expiration. From our findings we conclude that the scalenes, the parasternal intercostals and the diaphragm are mainly active during inspiration, and that the rectus abdominis muscle is predominantly active during expiration. These observations are consistant with the inspiratory or expiratory function of the muscles studied. This pattern remains the same in the tetraplegic patients examined.

Using magnetic transcranial motor cortex stimulation, we have been able to confirm the physiological findings of other authors based upon electromyography, transdiaphragmatic, oesophageal and/or abdominal pressure measurements, and changes in rib cage motion as evidenced by costal and sternal displacement. ${ }^{5,8-13,23,26,28}$

The MEP-latency times and CMCT in patients with incomplete tetraplegia were significantly $( \pm$ two standard deviations) prolonged in the scalenes and parasternal intercostals, but within normal limits for the diaphragm. This difference between the diaphragm and the other inspiratory muscles might be due to the multiple root innervation $(\mathrm{C} 3, \mathrm{C} 4$ and $\mathrm{C} 5)$ and the larger size of the diaphragm, as well as to the fact that the function of this muscle is more or less preserved. Moreover, the innervating nerve roots of the other inspiratory muscles are located at a lower medullary level ( $\mathrm{C} 4$ to $\mathrm{C} 8$ for the scalenes, and T2-T3 for the parasternal intercostals studied).

The MEP-amplitudes in all inspiratory muscles studied were significantly decreased in tetraplegic patients as compared to age-matched controls. This could in part be due to a decreased number of innervating axons and muscle hypotrophy.

No MEPs could be obtained from the abdominal muscles, except in one complete $\mathrm{C} 3$ tetraplegic patient, in whom only a very small response was seen during expiration, with a latency time of $26.9 \mathrm{~ms}$ (compared to $18.7 \pm 1.3 \mathrm{~ms}$ in controls) and an amplitude of $40 \mu \mathrm{V} \quad(886 \pm 440 \mu \mathrm{V}$ in controls). In summary, when MEPs were obtained from the abdominal muscles, their latency times were much more delayed than those of the inspiratory muscles and their amplitudes were considerably smaller. The much lower location of their innervating nerve roots (T10) and the much larger distance of their spinal exit zone from the level of injury at the cervical spinal cord might at least partially explain this phenomenon.

In the ventilator-dependent tetraplegic patient no MEPs could be obtained from any of the muscles studied. This was to be expected since this patient presented clinically motor complete, but sensory incomplete tetraplegia.

In conclusion, magnetic TCS is a painless and easily applicable technique to investigate the central motor conduction properties of the respiratory muscles, both in healthy humans and in patients with various neurological and respiratory disorders. Since the MEP-latency times and CMCT of the respiratory muscles were shown to have a high reproducibility and a very small variability, these variables can be used as a rigid parameter in the diagnosis and follow-up of these diseases, eg in tetraplegic subjects. 


\section{Acknowledgements}

The authors would like to thank J De Reuck, MD PhD and $\mathrm{E}$ Derom MD, PhD for their guidance and advice, as well as M Van Laere MD PhD chief of the Rehabilitation Center.

\section{References}

1 Bailar III JC, Mosteller F. Medical uses of statistics. New England Journal of Medicine Books 1986; pp 167-168.

2 Barker AT, Jalinous R, Freeston IL. Non-invasive stimulation of the human motor cortex. Lancet 1985; ii: 1106-1107.

3 Barker AT, Freeston IL, Garnham CW. Measurement of cortical and peripheral neural membrane time constants in man using magnetic nerve stimulation. J Physiol 1990; 423: 66P.

4 Buchtal F, Rosenfalck A, Behse F. Sensory potentials of normal and diseased nerve. In: Dyck PJ, Thomas PK, Lambert EH (eds). Peripheral neuropathy (2nd ed.), Saunders: Philadelphia, USA, 1984; vol. 1, pp $981-1015$.

5 Campbell EJM, Green JH. The expiratory function of the abdominal muscles in man. An electromyographic study. $J$ Physiol 1953; 120: $409-418$.

6 Cros D, Chiappa KH. Clinical applications of motor evoked potentials. In: Devinsky O, Beric A, Dogali M (eds). Electrical and magnetic stimulation of the brain and spinal cord. Raven Press: New York, 1993; pp 179-185.

7 Day BL, et al. Different sites of action of electrical and magnetic stimulation of the human brain. Neurosci Lett 1987; 75(1): $101-$ 106.

8 Delhez L. Contribution électromyograhique à l'étude de la mécanique et du côntrole nerveux des mouvements respiratoires de l'homme. Vaillant-Carmanne: Liège, Belgium, 1974.

9 De Troyer A, Bastenier J, Delhez L. Function of respiratory muscles during partial curarization in humans. J Appl Physiol 1980; 49: $1049-1056$.

10 De Troyer A, Sampson MG. Activation of parasternal intercostals during breathing efforts in human subjects. $J$ Appl Physiol 1982; 52: $524-529$.

11 De Troyer A, Estenne M. Coordination between rib cage muscles and diaphragm during quiet breathing in humans. J Appl Physiol 1984; 57(3): $899-906$.

12 De Troyer A, Ninane V. Triangularis sterni: a primary muscle of breathing in the dog. J Appl Physiol 1986; 60: 14-21.
13 De Troyer A, et al. Transversus abdominis muscle function in humans. J Appl Physiol 1990; 68: 1010-1016.

14 Dimitrijevic MR, et al. Early and late lower limb motor evoked potentials elicited by transcranial magnetic motor cortex stimulation. Electroenceph Clin Neurophysiol 1992; 85: 365-373.

15 Downie AW, Newell DJ. Sensory nerve conduction in patients with diabetes mellitus and control. Neurology 1961; 11: 876-882.

16 Gandevia SC, Rothwell JC. Activation of the human diaphragm from the motor cortex. J Physiol 1987; 384: 109-118.

17 Gandevia SC, Plassman BL. Responses in human intercostal and truncal muscles to motor cortical and spinal stimulation. Resp Physiol 1988; 73: $325-338$.

18 Gandevia SC, McKenzie DK, Plassman BL. Activation of human respiratory muscles during different manoeuvres. $J$ Appl Physiol 1990; 428: $387-403$.

19 Gardner MJ, Altman DG. Statistics with confidence. British Medical Journal (London), 1989, p 95.

20 Lipski J, Bektas A, Porter R. Short latency inputs to phrenic motoneurones from the sensorimotor cortex in the cat. Exp Brain Res 1986; 61: $280-290$.

21 Lissens MA (ed). Clinical applications of magnetic transcranial stimulation. Peeters Press: Leuven, Belgium, 1992.

22 Lissens MA. Motor evoked potentials of the human diaphragm elicited through magnetic transcranial brain stimulation. $J$ Neurol Sci 1994; 124: 204-207.

23 Lissens MA, et al. Motor evoked potentials of the abdominal muscles elicited through magnetic transcranial brain stimulation. Muscle Nerve 1995; 18: 1353 - 1354.

24 Mortola JP, Sant'Ambrogio G. Motion of the rib cage and the abdomen in tetraplegic patients. Clin Sci Mol Med 1978; 54: 25 32.

25 Oh SJ. Clinical Electromyography-Nerve Conduction Studies (2nd ed), Williams \& Wilkins: Baltimore, USA, 1993.

26 Raper AJ, et al. Scalene and sternomastoid muscle function. $J$ Appl Physiol 1966; 21: $497-502$.

27 Rothwell JC, et al. Stimulation of the human motor cortex through the scalp. Exp Physiol 1991; 76: 159-200.

28 Van der Schans CP, et al. Effect of positive expiratory pressure on breathing pattern in healthy subjects. Eur Respir J 1993; 6: $60-66$.

29 Zifko U, et al. Transcortical and cervical magnetic stimulation with recording of the diaphragm. Muscle Nerve 1996; 19: 614620 . 